\title{
An Improved Scheme on Morphological Image Segmentation Using the Gradients
}

\author{
Pinaki Pratim Acharjya \\ Assistant Professor, CSE dept \\ BITM, Santiniketan, \\ Bolpur, West Bengal
}

\author{
Santanu Santra \\ Assistant Professor, CSE dept \\ BITM, Santiniketan, \\ Bolpur, West Bengal
}

\author{
Dibyendu Ghoshal \\ Associate Professor, ECE dept \\ National Institute of Technology \\ Agartala, Tripura
}

\begin{abstract}
An improved scheme for contour detection with better performance measure has been proposed. It is based on the response of human visual system during visualization of any type of an image. The scheme consisted of two parts namely to find the edge of the image by using the modified mask of Laplacian of Gaussian edge operator and subsequent modulation of the edge by using watershed algorithm. The method has been applied to a digital image and better performance measure of contour detection has been achieved.
\end{abstract}

Keywords-Contour detection; gradients; watershed algorithm.

\section{INTRODUCTION}

Contour detection [1-3] in real life images is a major problem to enable them for subsequent processing by machines. Contours are salient coarse edge which belongs to object and image boundaries in the image. Saliency of an edge is a subjective matter [4] and the perception about the contour of an image varies from one human being to another one. Contours are sparser than the edges of an image as detected by various edge detector operators [2] and following various lower and upper threshold values for edge detection. Thus, it can be apprehended that contour map is an efficient representation of an image as it possesses only salient information and thus provide more valuable information for high level computer vision and recognition tasks. Hence design of improved contour detection scheme is gaining gradual importance.

To perform image segmentation and edge detection tasks, there are many detection techniques [13-22]. Among them in mathematical morphology watershed algorithm using gradients is a popular one. The initial stage of this segmentation [4-7] method is to produce a gradient image from the actual image. It has been observed that the use of standard 5x5 mask of Laplacian of Gaussian edge detector for image segmentation does not also solve the main problem associated with the watershed transform: over-segmentation. In this paper a modified scheme of Laplacian of Gaussian operator with 9x9 mask for generating gradient images is proposed and produces greater accuracy and lesser over segmentation [5-6] in edge detection with subsequent modulation of the edge by using watershed algorithm. The entropy which is a statistical measure of randomness that can be used to characterize the texture of the input image is studied along with peak signal to noise ratio (PSNR), mean square ratio (MSE) and execution times are also studied in this paper.
The structure of this work is the following: Section 2 introduces conventional Laplacian of Gaussian edge detection operator for gradient images. Section 3 presents a brief description on gradients. Section 4 and 5 is devoted to the segmentation process for edge detection using watershed algorithm. Section 6 presents the proposed scheme of modified Laplacian of Gaussian moderator with $9 \mathrm{x} 9$ mask. The results are discussed in section 7 and we finish this paper with some concluding remarks with section 8 .

\section{CONVENTIONAL LAPLACIAN OF GAUSSIAN OPERATOR}

This detector finds edges by looking for zero crossings after filtering $f(x, y)$ with a Laplacian of Gaussian filter. In this method, the Gaussian filtering is combined with Laplacian to break down the image where the intensity varies to detect the edges effectively. It finds the correct place of edges and testing wider area around the pixel. It have been observed and studied that the standard mask of Laplacian of Gaussian edge detector of 5x5mask can be modified and the scheme can be improved for generating masks of arbitrary size for gradient images for more accurate detections of object edges in a digital image. A 5x5 mask LOG filter has been shown in below.

\begin{tabular}{|c|c|c|c|c|}
\hline 0 & 0 & -1 & 0 & 0 \\
\hline 0 & -1 & -2 & -1 & 0 \\
\hline-1 & -2 & 16 & -2 & -1 \\
\hline 0 & -1 & -2 & -1 & 0 \\
\hline 0 & 0 & -1 & 0 & 0 \\
\hline
\end{tabular}

\section{MoRPHOLOGICALGRADIENT CALCULATION}

In contrast to classical area based segmentation, the watershed transform [2] was executed on the gradient image. A morphological gradient is the difference between the dilation and the erosion of a given image in mathematical morphology and digital image processing. It is an image where each pixel value (typically non- negative) indicates the contrast intensity in the close neighborhood of that pixel.

The morphological gradient $\mathrm{m}$ of a function $\mathrm{f}$ is defined by:

$$
m(p)=[(p \oplus s)-(p \ominus s)]
$$


Where, $(p \oplus s)(i)=\operatorname{Sup}(p(j))$ is the dilation of $f$ at the point $x$ and $(p \oplus s)(i)=\operatorname{Inf}(p(j))$ is the erosion of $f$ and $S$ would be the detection of obstacles but the main problem is structuring element applied on image.

\section{WATERSHED ALGORITHM}

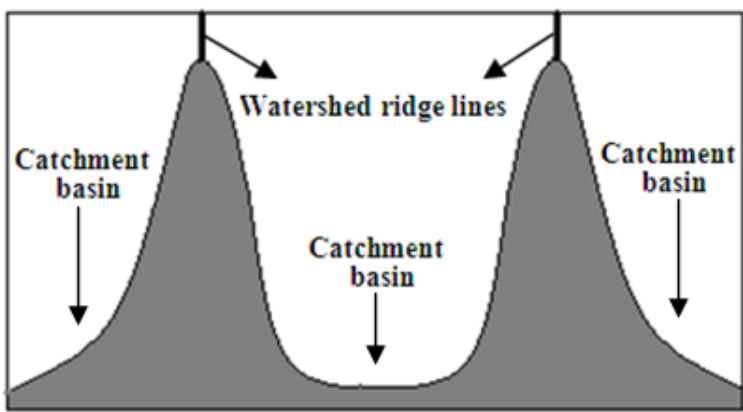

Fig. 1. Watershed segmentation-local minima yield catchment basins; local maxima define the watershed lines.

Watershed algorithm is a tool for morphological image segmentation. A gray scale image can be interpreted as the topographic image of landscape. This is accomplished (the image intensity) as an altitude. Using the features of these images, the technique of digital image processing called Watershed Transform. It consists in placing a water source in each regional minimum (catchment basins), to flood the relief from sources, and build barriers when different sources are meeting. The resulting set of barriers constitutes a watershed by flooding, i.e., the set of pixels along which the gray levels changes sharply gives rise to a watershed edge.

\section{MATHEMATICAL BACKGROUND OF WATERSHED ALGORITHM}

Assume, $M_{i}$ where $i=1$ to $\mathrm{n}$ be the set of coordinates points in the regional minima (catchment basins), of the image $P(x, y)$ and $C\left(M_{i}\right)$ be the coordinates points of catchment basins associated with the regional minima $M_{i}$

Where,

$$
T n=\{(s, t) \mid P(s, t)<n\}
$$

$T[n]=$ set of points in $P(x, y)$ which are lying below the plane $p(x, y)=n$

min, $\max =$ minimum or maximum gray level value.

$n=$ stage of flooding varies from $\min +1$ to $\max +1$

Let $C_{n}\left(M_{1}\right)$ be the set of points in the catchment basin associated with $M_{1}$ that are flooded at stage $\mathrm{n}$.

Where,

$$
\operatorname{Cn}(M 1)=\cap\{C(M 1), T[n]\}
$$

$$
\operatorname{Cn}\left(M_{i}\right)= \begin{cases}1, & \text { if }(x, y) \in C\left(M_{i}\right) \text { and }(x, y) \in T[n] \\ 0, & \text { otherwise }\end{cases}
$$

$\mathrm{C}[\mathrm{n}]$ is the union of flooded catchment basin portions at the stage $n$.

Where,

$$
\begin{aligned}
C[n] & =C n(m 1) \cup C n(m 2) \ldots \ldots C n(m R) \\
C[\max +1] & =C(m 1) \cup C(m 2) \ldots \ldots C(m R)
\end{aligned}
$$

If the algorithm keeps on increasing flooding level then $C_{n}\left(M_{i}\right)$ and $T[n]$ will either remain constant or increase. Algorithm initializes $C[\min +1]=T[\min +1]$, and then precedes recursively by assuming that at step $\mathrm{n} C[n-1]$ has been constructed.

Let, $G$ is a set of connected components in $T[n]$ and for each connected component $g \in G[n]$, there possibilities will arise.

1. $g \cap C[n-1]$ is empty. $-1]$.

2. $g \cap C[n-1]$ contains one connected component of $C[n$

3. $g \cap C[n-1]$ contains more than one connected component of $C[n-1]$.

\section{PROPOSED SCHEME}

In proposed scheme a modified 9x9 mask of Laplacian of Gaussian operator has been presented. After large number of trials using masks of larger dimensions, the size of the optimal mask was obtained and found to have $9 \times 9$ in dimensions. The modified mask is shown here.

\begin{tabular}{|c|c|c|c|c|c|c|c|c|}
\hline $\mathbf{0}$ & $\mathbf{0}$ & $\mathbf{0}$ & $\mathbf{1}$ & $\mathbf{1}$ & $\mathbf{1}$ & $\mathbf{0}$ & $\mathbf{0}$ & $\mathbf{0}$ \\
\hline $\mathbf{0}$ & $\mathbf{1}$ & $\mathbf{3}$ & $\mathbf{4}$ & $\mathbf{4}$ & $\mathbf{4}$ & $\mathbf{3}$ & $\mathbf{1}$ & $\mathbf{0}$ \\
\hline $\mathbf{0}$ & $\mathbf{2}$ & $\mathbf{3}$ & $\mathbf{2}$ & $\mathbf{2}$ & $\mathbf{2}$ & $\mathbf{3}$ & $\mathbf{2}$ & $\mathbf{0}$ \\
\hline $\mathbf{1}$ & $\mathbf{4}$ & $\mathbf{2}$ & $-\mathbf{8}$ & $-\mathbf{1 4}$ & $\mathbf{- 8}$ & $\mathbf{2}$ & $\mathbf{4}$ & $\mathbf{1}$ \\
\hline $\mathbf{1}$ & $\mathbf{4}$ & $\mathbf{2}$ & $-\mathbf{1 4}$ & $-\mathbf{3 0}$ & $-\mathbf{1 4}$ & $\mathbf{2}$ & $\mathbf{4}$ & $\mathbf{1}$ \\
\hline $\mathbf{1}$ & $\mathbf{4}$ & $\mathbf{2}$ & $\mathbf{- 8}$ & $-\mathbf{1 4}$ & $\mathbf{- 8}$ & $\mathbf{2}$ & $\mathbf{4}$ & $\mathbf{1}$ \\
\hline $\mathbf{0}$ & $\mathbf{2}$ & $\mathbf{3}$ & $\mathbf{2}$ & $\mathbf{2}$ & $\mathbf{2}$ & $\mathbf{3}$ & $\mathbf{2}$ & $\mathbf{0}$ \\
\hline $\mathbf{0}$ & $\mathbf{1}$ & $\mathbf{3}$ & $\mathbf{4}$ & $\mathbf{4}$ & $\mathbf{4}$ & $\mathbf{3}$ & $\mathbf{1}$ & $\mathbf{0}$ \\
\hline $\mathbf{0}$ & $\mathbf{0}$ & $\mathbf{0}$ & $\mathbf{1}$ & $\mathbf{1}$ & $\mathbf{1}$ & $\mathbf{0}$ & $\mathbf{0}$ & $\mathbf{0}$ \\
\hline
\end{tabular}

The flowchart of the proposed scheme is given in below. In initial stage a color image is converted into gray scale or black and white image. The gradient image is accrued from the grayscale image with the help of proposed modified mask of Laplacian of Gaussian edge detection operator. Finally the watershed algorithm is applied to detect the edges of the different objects within the image.




VII. Comparison Of Various Edge Detection OPERATORS WITH THE PROPOSED SCHEME

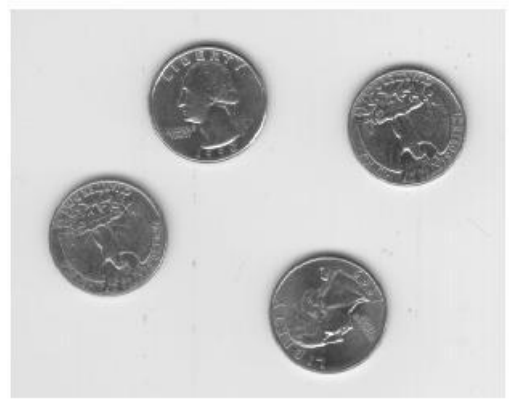

Fig. 2. Grayscale image

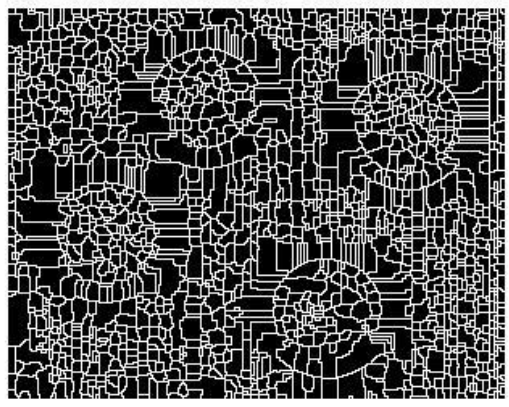

Fig. 3. Average.

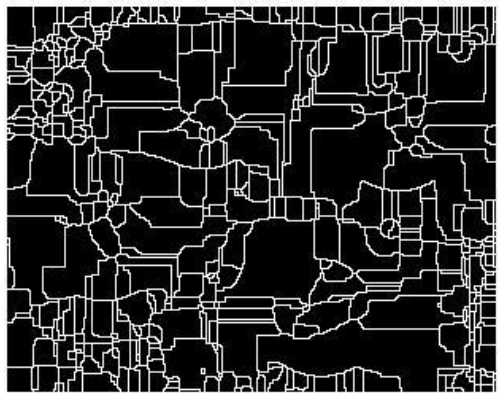

Fig. 4. Disk.

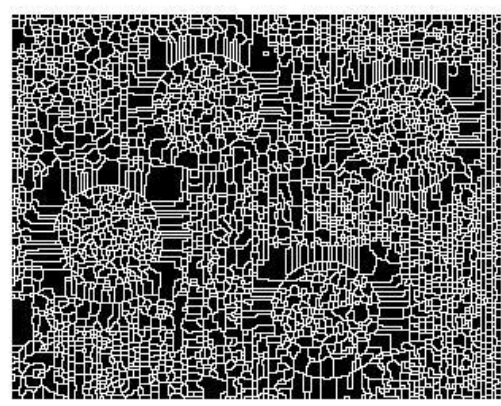

Fig. 5. Gaussian

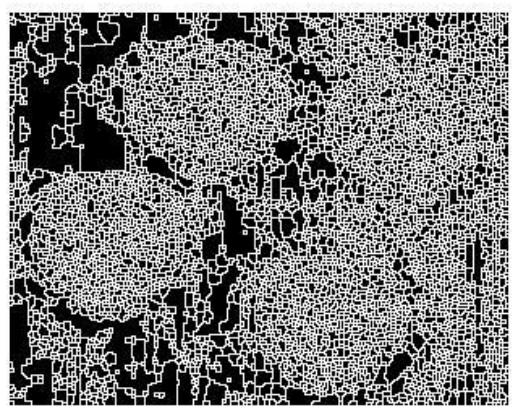

Fig. 6. Laplacian.

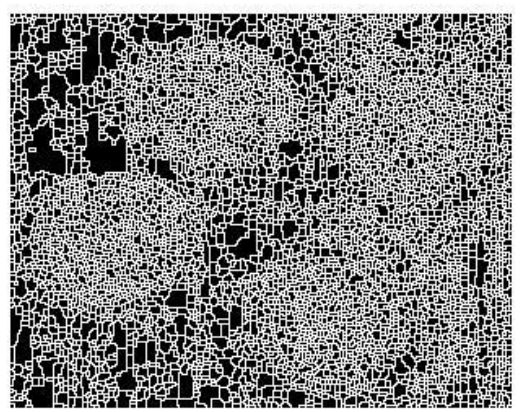

Fig. 7. Log. 


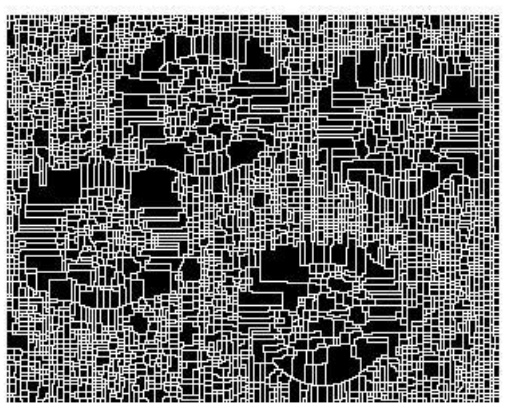

Fig. 8. Motion.

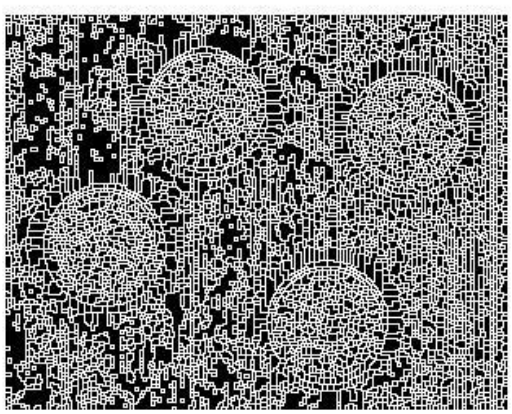

Fig. 9. Prewitt.

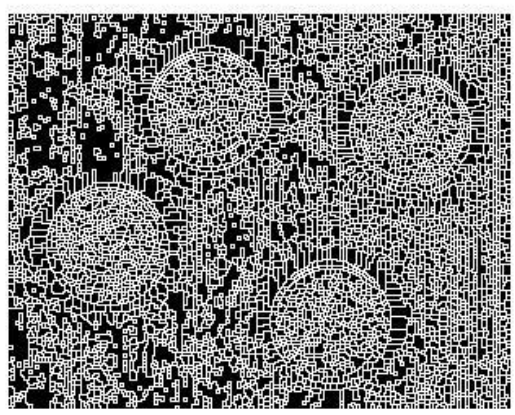

Fig. 10. Sobel.

The proposed approach is applied on a natural image and shown in from figure 2 to figure 12 respectively. Figure 2 shows the original image. Figure 3 to figure 11 illustrates the segmented images with watershed algorithm using different gradient operators like Average, Disk, Gaussian, Laplacian, Laplacian of Gaussian , Motion, Prewitt, Sobel and Unsharp. The segmented image using watershed algorithm with proposed scheme which is a modified 9x9 mask of Laplacian of Gaussian operator is shown in figure 12. We obtained output images that consist of all edge information and regions about the objects of input image.

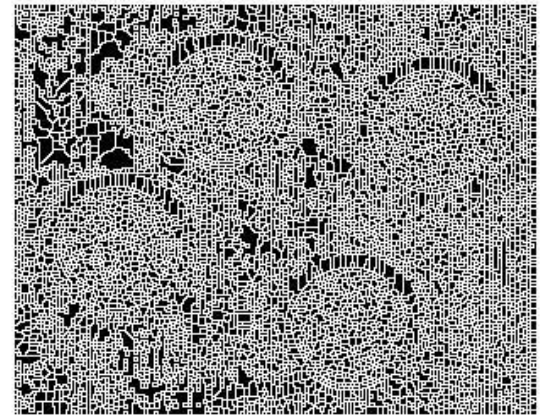

Fig. 11. Unsharp.



Fig. 12. Proposed scheme with modified 9x9 mask .

It have been observed by comparing the resultant images that the segmented images with watershed algorithm using conventional edge detection operators (figure 3 to figure 11) produces over segmentation and also the edges in the images are not very sharp. However the segmented image obtained by using the proposed scheme produces much better, accurate and sharp edges of different objects with less over segmentation. Statistical measurements of different segmented images are shown in table 1.

The entropies of the final segmented images using watershed algorithm through different edge detectors with watershed algorithm using gradients and the proposed scheme with modified log filter having 9x9 mask have been calculated and the values have been shown in the table 1 .

\section{CONCLUSION}

The present work introduced the concept of edge detection with gradients and has used it to produce an effective watershed segmentation technique for natural images. The resultant image with the proposed scheme produces much higher accuracy to detect object edges compared with the other segmented images that is obtained by applying watershed algorithm using different edge detection operators for generating gradients images. Additionally, an improved scheme on morphological image segmentation with gradients 
has been implemented for better and accurate edge detection counteracts the problem of over-segmentation.

TABLE I. STATISTICAL MEASUREMENT

\begin{tabular}{|l|l|l|l|}
\hline \multicolumn{1}{|c|}{ SEGMENTED IMAGE } & ENTROPY & PSNR & \multicolumn{1}{c|}{ MSE } \\
\hline Watershed with average & 2.5884 & 6.4471 & 14736 \\
\hline Watershed with disk & 2.2669 & 5.7638 & 17246 \\
\hline Watershed with Gaussian & 2.5808 & 6.8883 & 13312 \\
\hline Watershed with Laplacian & 2.5519 & 7.0469 & 12835 \\
\hline $\begin{array}{l}\text { Watershed with Laplacian of } \\
\text { Gaussian }\end{array}$ & 2.5527 & 7.2787 & 12168 \\
\hline Watershed with Motion & 2.5506 & 7.1162 & 12632 \\
\hline Watershed with Prewitt & 2.5752 & 7.2311 & 12302 \\
\hline Watershed with Sobel & 2.5662 & 7.2124 & 12355 \\
\hline Watershed with Unsharp & 2.5166 & 7.9300 & 10473 \\
\hline $\begin{array}{l}\text { Watershed with proposed } \\
\text { modified mask }\end{array}$ & 2.5910 & 6.8776 & 13345 \\
\hline
\end{tabular}

\section{REFERENCES}

[1] D. Ziou and S. Tabbone, "Edge detection techniques: An overview", International Journal of Pattern Recognition and Image Analysis, 8(4):537-559, 1998.

[2] W. Zhang and F. Bergholm, "Multi-scale blur estimation and edge type classification for scene analysis", International Journal of Computer Vision, vol 24, issue 3, Pages: 219 - 250, 1997.

[3] T. Pajdla and V. Hlavac, "Surface discontinuities in range images," in Proc IEEE 4th Int. Conf. Comput. Vision, pp. 524-528, 1993.

[4] K. Haris,"Hybrid image segmentation using watersheds and fast region merging," IEEE Trans Image Processing, 7(12), pp. 1684-1699, 1998.

[5] Vicent L. Solille P, Watershed in digital spaces, "An efficient algorithm based immersion simulations", IEEE Transections PAMI, pp. 538-598, 1991.

[6] S.Beucher, F.Meyer,-The morphological approach to segmentation: The watershed transform $\|$, in Mathematical Morphology Image Processing, E. R. Dougherty, Ed. New York Marcel Dekker, vol. 12, pp. 433-481, 1993

[7] Chen Pan, Congxun Zheng, Hao-Jun Wang, -Robust Color Image Segmentation Based On Mean Shift And Marker-controlled Watershed Algorithm ||, Second International Conference on Machine Learning and Cybernetics, 2-5 November 2003, Wan, pp. 2752-2756, 2003.
[8] Thilagamani, N.Shanthi, "A Novel Recursive Clustering Algorithm for Image Oversegmentation", European Journal of Scientific Research, Vol.52, No.3, pp.430-436, 2011.

[9] Peter Eggleston, "Understanding Oversegmentation and Region Merging", Vision Systems Design, December 1, 1998.

[10] P. Jackway, "Gradient watersheds in morphological scalespace," IEEE Trans. Image Processing vol. 15, pp. 913-921, June, 1996.

[11] Rafael C. Gonzalez, Richard E. Woods, Steven L. Eddins, "Digital Image Processing Using MATLAB," Second Edition, Gatesmark Publishing, 2009.

[12] Dubrovin, B.A.; A.T. Fomenko, S.P. Novikov, Modern Geometry-Methods and Applications: Part I: The Geometry of Surfaces, Transformation Groups, and Fields (Graduate Texts in Mathematics) (2nd ed.), Springer, pp. 14-17, 1991.

[13] S. Beucher, "Watershed, hierarchical segmentation and water fall algorithm," in Mathematical Morphology and Its Applications to Image Processing, Dordrecht, The Netherlands: Kluwer, 1994, pp. 69-76.

[14] Beucher, S., and Meyer, F. The morphological approach to segmentation: the watershed transformation. In Mathematical Morphology in Image Processing, E. R. Dougherty, Ed. Marcel Dekker, New York, ch. 12, pp. 433-481, 1993.

[15] Beucher, S., and Lantuejoul, C, "Use of watersheds in contour detection", In Proc. International Work-shop on Image Processing, RealTime Edge and Motion Detection/Estimation, Rennes, pp.17-21, France, September 1979.

[16] Vicent L. Solille P, Watershed in digital spaces, "An efficient algorithm based immersion simulations," IEEE Transections PAMI,vol. 13.no6. pp. 538-598, 1991.

[17] M. Couprie and G. Bertrand, "Topological grayscale watershed transformation," in Proc. SPIE Vision Geometry V, vol. 3168, pp. 136146, 1997

[18] C. Riddell, p. Brigger, R. E. Carson and S. L. Bacharach, "The watershed algorithm: a method to segment noisy PET transmission images," IEEE Trans. Nucl. Sci., vol. 46, no. 3, pp. 713-719, Mar, 1999.

[19] M. W. Hansen and W. E. Higgins, "Watershed-based maximumhomogeneity filtering," IEEE Trans. Image Process., vol. 8, no. 7, pp. 982-988, jul. 1999

[20] K. Haris,"Hybrid image segmentation using watersheds and fast region merging," IEEE Trans Image Processing, vol. 7, no. 12, pp. 1684-1699, 1998.

[21] F. Meyer, S. Beucher, "Morphological Segmentation," Journal of Visual Communication and Image Representation,vol. 1, pp. 21-46, 1990.

[22] D. Wang, "Unsupervised video segmentation based on waterseds and temporal traking," IEEE Trans. Circuits Syst. VideoTechnol., vol. 8, no. 5, pp. 539-546, May 1998. 